\title{
Simulating radiative astrophysical flows with the PLUTO code: a non-equilibrium, multi-species cooling function
}

\author{
O. Teşileanu, A. Mignone, and S. Massaglia \\ Dipartimento di Fisica Generale, Università degli Studi di Torino, via P. Giuria 1, 10125 Torino, Italy \\ e-mail: ovidiu.tesileanu@ph.unito.it
}

Received 25 January 2008 / Accepted 12 June 2008

ABSTRACT

\begin{abstract}
Context. Time-dependent cooling processes are of paramount importance in the evolution of astrophysical gaseous nebulae and, in particular, when radiative shocks are present. Given the recent improvements in resolution of the observational data, simulating these processes in a more realistic manner in magnetohydrodynamic (MHD) codes will provide a unique tool for model discrimination. Aims. The present work introduces a necessary set of tools that can be used to model radiative astrophysical flows in the optically-thin plasma limit. We aim to provide reliable and accurate predictions of emission line ratios and radiative cooling losses in astrophysical simulations of shocked flows. Moreover, we discuss numerical implementation aspects to ease future improvements and implementation in other MHD numerical codes.

Methods. The most important source of radiative cooling for our plasma conditions comes from the collisionally-excited line radiation. We evolve a chemical network, including 29 ion species, to compute the ionization balance in non-equilibrium conditions. The numerical methods are implemented in the PLUTO code for astrophysical fluid dynamics and particular attention has been devoted to resolve accuracy and efficiency issues arising from cooling timescales considerably shorter than the dynamical ones.

Results. After a series of validations and tests, typical astrophysical setups are simulated in 1D and 2D, employing both the present cooling model and a simplified one. The influence of the cooling model on structure morphologies can become important, especially for emission line diagnostic purposes.

Conclusions. The tests make us confident that the use of the presented detailed radiative cooling treatment will allow more accurate predictions in terms of emission line intensities and shock dynamics in various astrophysical setups.
\end{abstract}

Key words. radiation mechanisms: thermal - line: formation - ISM: Herbig-Haro objects - ISM: jets and outflows methods: numerical - magnetohydrodynamics (MHD)

\section{Introduction}

Astrophysical gases emit thermal radiation while undergoing dynamical transformations. There are cases where the gas is so diluted that the typical timescales for cooling greatly exceed the dynamical ones but, in many instances, cooling and the related ionization/recombination processes for the emitting species become comparable to, or faster than, the dynamical evolution of the system and should be considered. Classical examples of intensively radiating gases are $\mathrm{H}$ II regions, planetary nebulae, supernova remnants and star forming regions. Thus, when studying gas flows in such environments, particular care must be taken to treat the interplay between dynamics and radiation in the correct way. This is particularly true and crucial whenever radiative shocks are involved. When cooling is strong, the ionization fraction of the emitting species is far from that at equilibrium, but evolves so rapidly with time that it must be treated in a timedependent fashion.

Under these conditions, the magnetohydrodynamic (MHD) equations, coupled with the equations describing the evolution of the emitting species and the radiative losses, must be solved by numerical means. The numerical problems posed by radiative cooling are particularly challenging whenever the advance time step of the integration is controlled by radiation/ionization rather than dynamics. This may happen in few grid points in the computational domain where the radiative losses are intense, e.g., right behind a shock front, where the cooling time becomes very small. Therefore, it is necessary to devise strategies able to deal with very different integration timescales: this is one of the points we address.

Time dependent ionization calculations were previously performed for gaseous nebulae by Marten \& Szczerba (1997) in hydrostatic conditions. Their approach is similar to our implementation of the ionization state treatment, however the ion species and implemented physical processes are in part different. Also, radiative cooling in optically-thin plasmas was previously investigated, and synthetic cooling functions were designed (e.g., Schmutzler \& Tscharnutter 1993). Among the radiative numerical codes employed in astrophysics, one can quote the hydrocode YGUAZUं (Raga et al. 2000), ASTROBEAR MHD code (Poludnenko et al. 2005; Berger \& LeVeque 1998) and Virginia Hydrodynamics - 1 (VH-1, Sutherland et al. 2003; Blondin \& Lufkin 1993). The MHD simulation code we use for our astrophysical applications - PLUTO - is a freely distributed application developed and maintained at the Turin University Turin Astronomical Observatory (Mignone et al. 2007). A previous numerical analysis about the evolution of radiative shocks in Young Stellar Object (YSO) jets (Massaglia et al. 2005) was carried out with PLUTO, using a simplified model for the radiative cooling losses, which evolved in time only the ionization fraction of hydrogen (cf., Rossi et al. 1997). This model will be called from now on SNEq (Simplified Non-Equilibrium cooling). 
We illustrate a more general treatment of atomic cooling and evolution of the ionization fraction of the emitting species, embedded in the PLUTO code as well, for use within MHD simulations of astrophysical interest. We will call this new cooling function MINEq (Multi-Ion Non-Equilibrium cooling). The main advantage of our approach is the full ionization state computation during the MHD simulation, which allows for better predictions of emission line intensities.

Section 2 contains a general overview of the adopted method and implementation of the treatment of radiative losses. Then, in Sect. 3, a description of the physics of the cooling model can be found, followed in Sect. 4 by the validations and tests in equilibrium conditions. The numerical implementation and testing are discussed in Sect. 5, while in Sect. 6 we present some typical astrophysical applications. Technical details on ionizationrecombination processes and numerical issues are presented in extended form in the Appendix.

\section{General overview}

The general characteristics and application ranges of the new cooling function added to the PLUTO code are summarized below. The density limits are those typically encountered in clouds and YSO jets, while the temperature range is limited by the highest ionization stage considered (at the high end) and the lack of molecular cooling (at the low end):

$N \in\left(10^{-2}, 10^{5}\right) \mathrm{cm}^{-3}, \quad T \in\left(2 \times 10^{3}, 2 \times 10^{5}\right) \mathrm{K}$.

However, the module is designed to permit later extension in terms of applicable parameter range (through adding more ion species, or a tabulated cooling function for higher temperatures) and physical processes taken into consideration.

Flow variables such as density $\rho$, velocity $\boldsymbol{v}$, magnetic field $\boldsymbol{B}$, and total energy $E$ are evolved according to the standard MHD equations:

$$
\begin{aligned}
& \frac{\partial \rho}{\partial t}+\nabla \cdot(\rho \boldsymbol{v})=0 \\
& \frac{\partial(\rho \boldsymbol{v})}{\partial t}+\nabla \cdot\left(\rho \boldsymbol{v} \boldsymbol{v}^{\mathrm{T}}-\boldsymbol{B} \boldsymbol{B}^{\mathrm{T}}+I p_{\mathrm{t}}\right)=0 \\
& \frac{\partial \boldsymbol{B}}{\partial t}-\nabla \times(\boldsymbol{v} \times \boldsymbol{B})=0 \\
& \frac{\partial E}{\partial t}+\nabla \cdot\left[\left(E+p_{\mathrm{t}}\right) \boldsymbol{v}-(\boldsymbol{v} \cdot \boldsymbol{B}) \boldsymbol{B}\right]=S_{E},
\end{aligned}
$$

where $S_{E}$ (described later) is a radiative loss term, and $p_{\mathrm{t}} \equiv$ $p+|\boldsymbol{B}|^{2} / 2$ denotes the total pressure (thermal + magnetic) of the fluid. We assume an ideal equation of state by which the total energy density becomes

$E=\frac{p}{\Gamma-1}+\rho \frac{|\boldsymbol{v}|^{2}}{2}+\frac{|\boldsymbol{B}|^{2}}{2}$,

with $\Gamma=5 / 3$ being the specific heats ratio.

The cooling model accounts for the evolution of 29 ion species, namely: HI, H II, He I and He II, and the first five ionization stages of $\mathrm{C}, \mathrm{N}, \mathrm{O}, \mathrm{Ne}$ and $\mathrm{S}$. Sulphur, although not having an important contribution to cooling, is added for diagnostic purposes (line ratios). The ionization network employed is larger than in most other MHD codes.

For each ion, we solve the additional equation

$$
\frac{\partial\left(\rho X_{\kappa, i}\right)}{\partial t}+\nabla \cdot\left(\rho X_{\kappa, i} \boldsymbol{v}\right)=\rho S_{\kappa, i}
$$

coupled to the original system of conservation laws (2)-(5). In Eq. (7) and throughout the following, the first index $(\kappa)$ corresponds to the element, while the second index $(i)$ corresponds to the ionization stage. Specifically, $X_{\kappa, i} \equiv N_{\kappa, i} / N_{\kappa}$ is the ion number fraction, $N_{\kappa, i}$ is the number density of the $i$ th ion of element $\kappa$, and $N_{K}$ is the element number density. We denote the whole set of ions for all possible $\kappa$ and $i$ with $\boldsymbol{X} \equiv\left\{X_{\kappa, i}\right\}$.

The source term $S_{\kappa, i}$ accounts for ionization and recombination and will be described in the following. The total line emission from the considered species enters in the source term $S_{E}$ in Eq. (5) and should give a good approximation of radiative cooling for the above conditions (Raga et al. 1997).

The system of Eqs. (2)-(5) together with (7) is integrated using the PLUTO code for computational astrophysics (Mignone et al. 2007). We take advantage of operator splitting techniques, where the homogeneous part of the equations (i.e., with $S_{\kappa, i}=$ $S_{E}=0$ ) is solved separately from the source step. The order of the respective advection and source operators $\left(\mathcal{H}^{\Delta t^{n}}\right.$ and $\left.\mathcal{S}^{\Delta t^{n}}\right)$ is reversed every step by keeping the time step $\Delta t^{n}=\Delta t^{n+1}$ constant for two consecutive integrations to guarantee formal second order accuracy. Thus, if $\boldsymbol{U}=\{\rho, \rho \boldsymbol{v}, \boldsymbol{B}, E, \rho \boldsymbol{X}\}$ is the vector of conserved variables, the solution advances from $t^{n}$ to $t^{n}+\Delta t^{n}$ as

$\boldsymbol{U}\left(t^{n}+\Delta t^{n}\right)=\mathcal{S}^{\Delta t^{n}} \mathcal{H}^{\Delta t^{n}} \boldsymbol{U}\left(t^{n}\right)$,

and from $t^{n}+\Delta t^{n}$ to $t^{n}+2 \Delta t^{n}$ as

$\boldsymbol{U}\left(t^{n}+2 \Delta t^{n}\right)=\mathcal{H}^{\Delta t^{n}} \mathcal{S}^{\Delta t^{n}} \boldsymbol{U}\left(t^{n}+\Delta t^{n}\right)$.

A new time step, $\Delta t^{n+2}$, is then computed as shown in Sect. 5 .

\section{Cooling module description}

We will restrict our attention to the source step only and remind the interested reader of the original paper by Mignone et al. (2007) for implementation details on the solution of the homogeneous MHD equations.

During the source step, in virtue of operator splitting, only internal energy $p /(\Gamma-1)$ and ion fractions $X_{\kappa, i}$ are affected. Density, velocity, and magnetic fields remain constant with the values provided by the most recent step. Thus Eqs. (5) and (7) are treated as a system of ordinary differential equations (ODE):

$\frac{\mathrm{d}}{\mathrm{d} t}\left(\begin{array}{c}p \\ X_{\kappa, i}\end{array}\right)=\left(\begin{array}{c}(\Gamma-1) S_{E} \\ S_{\kappa, i}\end{array}\right)$,

where $\kappa=\mathrm{H}, \mathrm{He}, \mathrm{C}, \ldots$ labels the element and $i=\mathrm{I}, \mathrm{II}, \mathrm{III}, \ldots$ identifies the ionization stage. Equations (10) must be solved for a time increment $\Delta t^{n}$ with initial condition provided by the output of the previous step (i.e., either an advection or source one).

Pressure $p$ and temperature $T$ are related by the ideal gas equation:

$p=N k_{\mathrm{B}} T \quad$ with $\quad N=\frac{\rho}{m_{\mathrm{u}} \mu(\boldsymbol{X})}$,

where $N$ is the total particle (atoms + electrons) number density, $k_{\mathrm{B}}$ is the Boltzmann constant, $m_{\mathrm{u}}$ is the atomic mass unit, and $\mu(\boldsymbol{X})$ is the mean molecular weight:

$\mu(\boldsymbol{X})=\frac{\sum_{\kappa} m_{\kappa} B_{\kappa}}{\sum_{\kappa} \sum_{i} X_{\kappa, i} \gamma_{i} B_{\kappa}}$

In Eq. (12) $m_{\kappa}$ is the atomic mass (in units of $m_{\mathrm{u}}$ ) of element $\kappa$ and $\gamma_{i}$ denotes the number of the ionization stage in spectroscopic notation for each ion. $B_{K}$ is the fractional abundance of the element. 


\subsection{Radiative losses}

Radiative losses are described by the source term $S_{E}$ in the energy Eq. (5):

$S_{E}=-\left(N_{\mathrm{at}} N_{\mathrm{el}} \Lambda(T, \boldsymbol{X})+L_{\mathrm{FF}}+L_{\mathrm{I}-\mathrm{R}}\right)$,

where $\Lambda(T, X)$ is the radiative cooling function due to collisionally-excited line radiation, $L_{\mathrm{FF}}$ denotes the free-free (bremsstrahlung) losses from $\mathrm{H}^{+}$and $\mathrm{He}^{+}$, while $L_{\mathrm{I}-\mathrm{R}}$ accounts for the energy lost during ionization/recombination processes. The number densities $N_{\text {at }}$ and $N_{\mathrm{el}}$ are, respectively, the total atom and electron number densities, readily determined from the mass density and the known chemical composition of the plasma (by default supposed solar, but customizable by the user):

$N_{\mathrm{at}}=\sum_{\kappa} N_{\kappa}$,

$N_{\mathrm{el}}(\boldsymbol{X})=N \sum_{\kappa} \sum_{i} X_{\kappa, i}\left(\gamma_{i}-1\right) B_{\kappa}$

Note that $N_{\text {at }}$ does not depend on the ionization state of the elements and it should not be confused with $N$, the total particle (atoms + electrons) number density used in the equation of state (11).

Emission lines from Fe II, Si II, and $\mathrm{Mg}$ II that exist in SNEq are added empirically to the energy losses of MINEq (without evolving the respective ion species) because of their importance at low temperatures.

\subsubsection{Energy loss by collisionally-excited line radiation}

The main contribution to radiative cooling comes from collisional excitation of low-lying energy levels of common ions, such as $\mathrm{O}$ and $\mathrm{N}$. In spite of their low abundances, these ions make a significant contribution because they have energy levels with excitation potentials of the order of $k T$. The total radiative cooling function $\Lambda(T, X)$ used in the energy source term (Eq. (13)) is:

$\Lambda(T, \boldsymbol{X})=\sum_{\kappa} \sum_{i} X_{\kappa, i} \mathcal{L}_{\kappa, i}\left(N_{\mathrm{el}}, T\right) B_{K}$,

where the sums are extended to all ion species and $B_{K}$ is the fractional abundance of the element $\kappa$.

Individual contributions to the different $\mathcal{L}^{\prime} \mathrm{s}^{1}$ are given by

$\mathcal{L}=\sum_{j} \hat{N}_{j} \sum_{l<j} A_{j l} h v_{j l}$

where $\hat{N}_{j}$ is the population of the $j$ th excitation level; $A_{j l}$ are the Einstein A coefficients; and $v_{j l}$ the emission line frequency for a transition between levels $j$ and $l$. We consider a 5-level atom model to compute the line radiation (Osterbrock \& Ferland 2005) by solving for the equilibrium populations in each of the excitation levels $j=1 \ldots 5$ :

$\sum_{l \neq j} \hat{N}_{l} N_{\mathrm{el}} q_{l j}+\sum_{l>j} \hat{N}_{l} A_{l j}=\sum_{l \neq j} \hat{N}_{j} N_{\mathrm{el}} q_{j l}+\sum_{l<j} \hat{N}_{j} A_{j l}$,

which, together with the normalization condition for the total number density of the ion, $\sum_{j} \hat{N}_{j}=N_{\kappa, i}$, can be solved for the relative $\hat{N}_{j}$ populations in each level.

\footnotetext{
1 In this section only, $\kappa$ and $i$ will be omitted unless necessary to avoid cluttered notation.
}

The 5-level atom model provides the great majority of the emission lines for the considered range of temperatures and thus gives a reliable estimation of the total line cooling.

For most of the ion species, the emission coefficients were taken from Pradhan \& Zhang (1999). The data for hydrogen was taken from Giovanardi et al. (1987) and their fit formula. The C II data comes from Blum \& Pradhan (1992), while for N II and N III Chebyshev polynomial fits from Stafford et al. (1994) were used.

\subsubsection{Free-free radiation}

A minor contributor to the cooling rate at moderate temperatures is the bremsstrahlung (free-free) radiation, having a continuous spectrum. The rate of cooling in this process by ions of charge $Z$, integrated over frequency, is approximately (Osterbrock \& Ferland 2005)

$L_{\mathrm{FF}}=1.42 \times 10^{-27} Z^{2} T^{1 / 2} N_{\mathrm{el}} N_{+}$,

in erg $\mathrm{cm}^{-3} \mathrm{~s}^{-1}$. Because of its abundance, $\mathrm{H}^{+}$dominates the free-free cooling, and $\mathrm{He}^{+}$can be included along with it since both have the same charge: $N_{+} \equiv N_{\mathrm{HII}}+N_{\mathrm{HeII}}$.

\subsubsection{Ionization-recombination losses}

Thermal energy is absorbed by the atom to pass to the next ionization stage. During the recombination, a free electron is captured and a part of its thermal energy is lost. The ionization/recombination losses are treated similarly in MINEq and SNEq, following the method described in Rossi et al. (1997):

$$
\begin{aligned}
& L_{\mathrm{I}-\mathrm{R}}=L_{\mathrm{I}}+L_{\mathrm{R}} \\
& L_{\mathrm{I}}=1.27 \times 10^{-23} \sqrt{T} N_{\mathrm{HI}} \mathrm{e}^{-\frac{157890}{T}} N_{\mathrm{el}} \\
& L_{\mathrm{R}}=2.39 \times 10^{-27} \sqrt{T} N_{\mathrm{HII}} N_{\mathrm{el}}
\end{aligned}
$$

expressed in erg $\mathrm{cm}^{-3} \mathrm{~s}^{-1}$.

The complementary effects of these processes on the plasma (the creation/destruction of a free particle) are accounted through the mean molecular weight, which varies together with the total particle number density.

\subsection{Ionization network}

Our ionization network can be written in terms of the sourceterm $S_{\kappa, i}$ mentioned above (Eq. (10)):

$S_{\kappa, i}=N_{\mathrm{el}}\left[X_{\kappa, i+1} \alpha_{\kappa, i+1}-X_{\kappa, i}\left(\zeta_{\kappa, i}+\alpha_{\kappa, i}\right)+X_{\kappa, i-1} \zeta_{\kappa, i-1}\right]$

where $\zeta_{\kappa, i}$ and $\alpha_{\kappa, i}$ are the ionization and recombination coefficients of the $i$ th ion specie of the element $\kappa$, defined as follows:

$\zeta_{\kappa, i}=\zeta_{\kappa, i}^{\mathrm{coll}}(T)+\frac{N_{\mathrm{HII}}}{N_{\mathrm{el}}} \zeta_{\kappa, i}^{\mathrm{HII}}(T)$

$\alpha_{\kappa, i}=\alpha_{\kappa, i}^{\mathrm{el}}(T)+\frac{N_{\mathrm{HI}}}{N_{\mathrm{el}}} \alpha_{\kappa, i}^{\mathrm{HI}}(T)+\frac{N_{\mathrm{HeI}}}{N_{\mathrm{el}}} \alpha_{\kappa, i}^{\mathrm{HeI}}(T)$,

where $N_{\mathrm{HII}} \equiv N_{\mathrm{H}} X_{\mathrm{HII}}=N_{\mathrm{at}} B_{\mathrm{H}} X_{\mathrm{HII}}$ is the number density of protons, $N_{\mathrm{HI}}$ and $N_{\mathrm{HeI}}$ are the number densities of neutral hydrogen and helium, respectively. The $\alpha_{\kappa, i}$ and $\zeta_{\kappa, i}$ coefficients are the transition rates corresponding to the reaction mechanisms defined in the Appendix A (note that $\alpha_{\kappa, i}^{\mathrm{el}}$ is the total electronion recombination coefficient, that is dielectronic plus radiative, $\left.\alpha^{\mathrm{el}}=\alpha^{\mathrm{DR}}+\alpha^{\mathrm{RR}}\right)$. 
Since we only consider part of the ions from each element (up to the fourth level of ionization, except for $\mathrm{H}$ and $\mathrm{He}$ ), the ionization rate for the highest state will be set to zero. This will produce saturation of the ion population in this state at very high temperatures, and limit the applicability of the cooling function. The temperature range can however be extended by adding further ionization stages for the elements.

For efficiency purposes, the ionization and recombination coefficients on the right-hand side of Eqs. (22) and (23) are sampled at discrete values of temperature at the beginning of integration and used as lookup tables.

\section{Comparison with equilibrium models}

We perform theoretical line ratios tests to verify the collision strengths in the radiative losses. Also, the total cooling function for an equilibrium ionization balance function of temperature (the effective cooling curve) was tested and found to be consistent with results obtained with more complex models.

\subsection{Equilibrium ionization balance}

The equilibrium ionization balance may be used as an initial condition for numerical simulations, and also serves for testing the ionization/recombination coefficients employed in the ionization network.

The ionization balance for each element at equilibrium is computed by setting $\mathrm{d} X_{\kappa, i} / \mathrm{d} t=0$ (for all ions) in Eq. (10). The equation for the highest ionization stage is replaced by the normalization condition,

$$
\sum_{i=1}^{K} X_{\kappa, i}=1
$$

where $K$ is the highest ionization state taken in consideration for the element $\kappa$. Thus, for each element, we solve the following system of equations:

$X_{\kappa, i+1} \alpha_{\kappa, i+1}-X_{\kappa, i}\left(\zeta_{\kappa, i}+\alpha_{\kappa, i}\right)+X_{\kappa, i-1} \zeta_{\kappa, i-1}=0$,

with $i=1, \ldots, K-1$ complemented by Eq. (24). Despite its aspect, the previous system of equations is not linear since the $\zeta$ and $\alpha$ coefficients depend on the concentrations themselves (see Eqs. (22) and (23)), so an iterative procedure must be employed to converge to the correct solution.

In the particular cases of hydrogen and helium, because of the charge-transfer reactions they are involved in, an exact treatment would also force $\zeta$ and $\alpha$ to depend on the number densities of all other ions that take part in these processes. Considering the very limited influence of these reactions on the hydrogen and helium ionization balance, we chose to neglect such influences.

Given an initial guess on the ionization state of the plasma, the systems of equations for equilibrium are solved, providing new values of $N_{\mathrm{el}}, N_{\mathrm{HI}}$, and $N_{\mathrm{HeI}}$. The process is repeated until the differences between the old and the new solutions are below a certain threshold. The convergence is rapidly achieved, generally less than five iterations are needed for a $10^{-4}-10^{-3}$ relative threshold. This is acceptable, considering that this equilibrium computation is typically done on the whole computation grid only once, in the beginning of the simulation. In Fig. 1, we show the equilibrium ionization balance as a function of temperature for three selected elements. Our results favourably compare to those obtained by previous investigators - such as Sutherland \& Dopita (1993) - with the ionization fractions being within $5-10 \%$ at the same temperature.

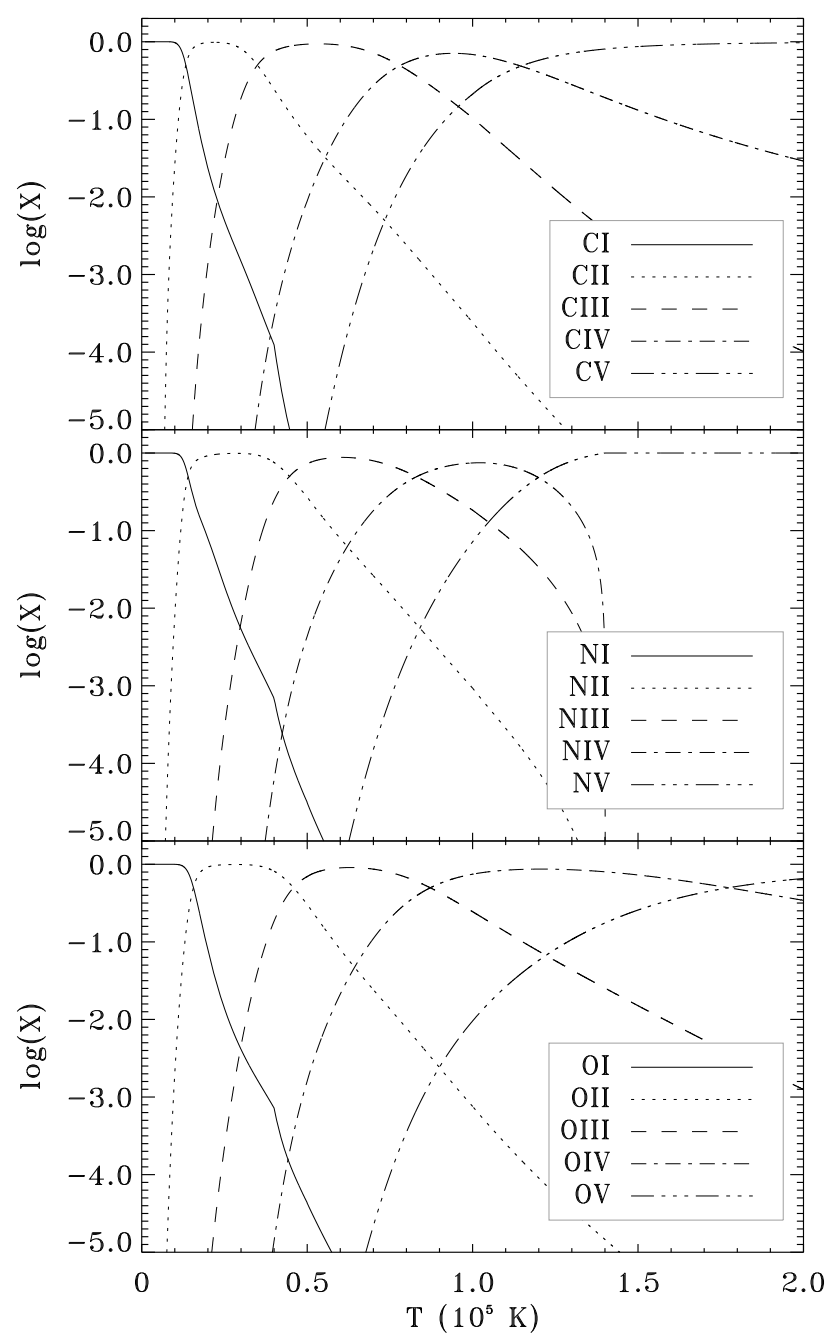

Fig. 1. Ionization fractions at equilibrium for the five ionization levels considered for C (top panel), $\mathrm{N}$ (middle panel), and $\mathrm{O}$ (bottom panel).

\subsection{Line ratios tests}

These tests are useful to verify the emission lines data and the level population computation routine (in our 5-level atom model). An example is presented here.

A popular way of estimating the temperatures in gaseous nebulae is to use the ratio of spectral line intensities, such as the lines of O III:

$$
\frac{\epsilon(\lambda 5007)+\epsilon(\lambda 4959)}{\epsilon(\lambda 4363)}=\frac{\epsilon\left({ }^{1} D_{2} \rightarrow{ }^{3} P_{2}\right)+\epsilon\left({ }^{1} D_{2} \rightarrow{ }^{3} P_{1}\right)}{\epsilon\left({ }^{1} S_{0} \rightarrow{ }^{1} D_{2}\right)}
$$

Inserting numerical values of the collision strengths and transition probabilities (Osterbrock \& Ferland 2005), the ratio becomes:

$R=\frac{\epsilon(\lambda 5007)+\epsilon(\lambda 4959)}{\epsilon(\lambda 4363)}=\frac{8.32 \exp \left(\frac{3.29 \times 10^{4}}{T}\right)}{1+4.5 \times 10^{-4} \frac{N_{\mathrm{el}}}{T^{1 / 2}}}$

for temperatures around $10000 \mathrm{~K}$.

Line ratios computed with the previous formula and the results of the 5-level atom model were compared, the differences of less than $\approx 6 \%$ being due to the fact that our code uses temperature-dependent collision strengths, while in the formula above they are assumed constant. 


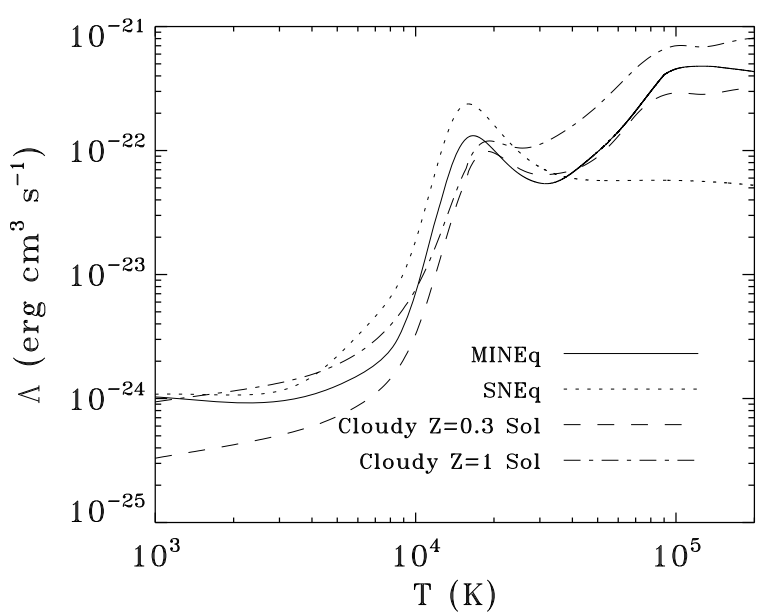

Fig. 2. Effective cooling curves in the temperature range from $10^{3}$ to $2 \times$ $10^{5} \mathrm{~K}$, comparison between the results obtained with MINEq, SNEq, and Cloudy.

\subsection{Effective cooling}

In Fig. 2, we plot the effective cooling function (in erg $\mathrm{cm}^{3} \mathrm{~s}^{-1}$ ) using the ionization fractions computed at equilibrium. For the sake of comparison, we also show the results obtained with the SNEq model described in Rossi et al. (1997) and the Cloudy atomic code, which has a large chemical network (see Ferland et al. 1998), for similar plasma conditions. Solar abundances have been assumed for all cooling functions, except for the Cloudy $Z=0.3$ case (where the metallicity is only 0.3 times the solar one). The SNEq model consists of the emission of 17 most important lines, plus the two-photon continuum and the radiative losses from ionization/recombination processes. In this model, however, only the ionization of $\mathrm{H}$ is evolved with the integration, the rest of the ions abundances being fixed or locked by charge-transfer processes to the ionization state of $\mathrm{H}$.

The results show a good agreement between the newlydeveloped cooling model (MINEq) and the computations carried out with the Cloudy code. Chemical composition (more extended in Cloudy that is an atomic code) and physical processes considered account for the differences. The MINEq effective cooling, considering only few metals, generally lies in between the results obtained with the Cloudy code for $Z=0.3 Z_{\odot}$ and $Z=Z_{\odot}$. The faster increase in the peak at $17000 \mathrm{~K}$ due to hydrogen Ly $\alpha$ presented by MINEq is due to the different sources of the ionization/recombination and emission coefficients (collision strengths).

It can be inferred that while MINEq accounts with good accuracy for the cooling losses up to $2 \times 10^{5} \mathrm{~K}$, SNEq cannot follow them above $3 \times 10^{4} \mathrm{~K}$ because it lacks higher ionization stages for the atoms. Furthermore, the effective cooling obtained with the MINEq model closely reproduces the early work of Dalgarno \& McCray (1972) in the temperature range considered.

\section{Numerical implementation}

In the source step, we advance the system of ordinary differential equations (ODE) given by Eq. (10) in each computational zone. For ease of notations, we rewrite the system as

$\frac{\mathrm{d} y}{\mathrm{~d} t}=\boldsymbol{f}(\boldsymbol{y})$,

where $\boldsymbol{y} \equiv\left\{p, X_{\kappa, i}\right\}$ and $\boldsymbol{f} \equiv\left\{(\Gamma-1) S_{E}, S_{\kappa, i}\right\}$ are, respectively, the vector of unknowns and right-hand sides for all possible values

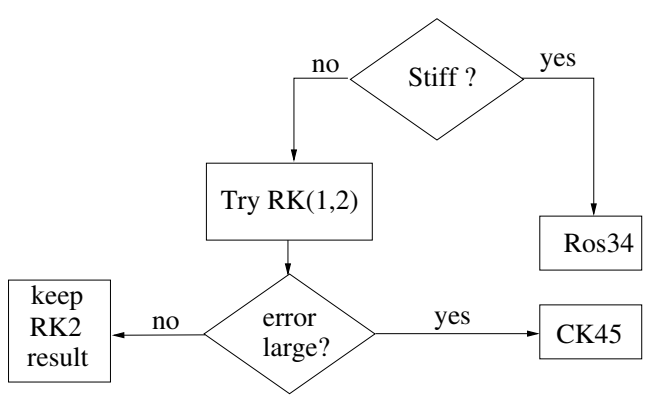

Fig. 3. Flowchart of the dynamically switching integration algorithm for cooling: Runge-Kutta 12, Rosenbrock 34, and Cash-Karp 45. Stiffness is detected according to Eq. (29).

of $\kappa$ and $i$ in a given computational cell. According to the notations introduced in Eqs. (8) and (9), we write the formal solution to (28) for a time increment $\Delta t^{n}$ as $\boldsymbol{y}^{*}=\mathcal{S}^{\Delta t^{n}} \boldsymbol{y}^{0}$, where the initial condition $\boldsymbol{y}^{0}$ is given by the output of the previous step.

\subsection{Integration strategy}

Accurate numerical integrations of Eq. (28) should be carried out consistently with the different timescales that may concurrently co-exist, according to the initial density, temperature and chemical concentrations. In addition, the system evolution dictated by the local ionization, recombination, and cooling rates may proceed considerably faster than the typical time scale imposed during the advection step. Under some circumstances, this contrast may lead to a stiff system of ODE. A common occurrence takes place, for instance, when a strong shock propagates in a cold neutral medium: as the front advances from one computational cell to the next, the ion populations will try to re-adjust to the sudden increase in temperature at a rate given by the ionization coefficients. At high temperatures $\left(T \gtrsim 2 \times 10^{4}\right)$, this process may proceed more and more rapidly.

Nevertheless, these kinds of events are extremely localized in space since most regions ahead of and far behind the shock wave are either close to equilibrium or evolve on much slower recombination scales. This suggests some form of selective integration by which regions of the flow undergoing very rapid changes should be promptly detected and treated accordingly. We achieve this by 1) detecting potential "stiffness" due to large ionization and recombination coefficients given by Eqs. (22), (23) and 2) monitoring, in each computational cell, the accuracy through an estimate of the local truncation error. We now describe in detail the numerical implementation of a dynamically-adaptive integration strategy, also shown in Fig. 3.

At the beginning of integration, we tag a computational cell as "non-stiff" if the integration time step satisfies

$\Delta t^{n}<\frac{1}{N_{\mathrm{el}} \max _{\kappa, i}\left(\left|\zeta_{\kappa, i}+\alpha_{\kappa, i}\right|\right)}$

where $N_{\mathrm{el}}$ is computed in the considered cell. If the previous condition holds ${ }^{2}$, we solve Eq. (28) using an explicit method with adaptive stepsize control. Embedded Runge-Kutta (RK) pairs simultaneously giving solutions of order $m$ and $m-1$ are preferred, since they provide an efficient error estimate. The most simple $(2,1)$ pair $(m=2)$, for example, may be obtained using a simple

\footnotetext{
2 This is, in fact, half the stability limit for the 1st order explicit Euler method.
} 
combination of two right-hand side evaluations yielding, respectively, 1 st- and 2 nd-order accurate solutions $\boldsymbol{y}_{1}$ and $\boldsymbol{y}_{2}$ :

$\boldsymbol{y}^{1}=\boldsymbol{y}^{0}+\Delta t^{n} \boldsymbol{f}\left(\boldsymbol{y}^{0}\right)$

$\boldsymbol{y}^{2}=\boldsymbol{y}^{0}+\Delta t^{n} \boldsymbol{f}\left(\boldsymbol{y}^{0}+\frac{\Delta t^{n}}{2} \boldsymbol{f}^{0}\right)$

The difference between the two solutions, $\boldsymbol{y}^{1}$ and $\boldsymbol{y}^{2}$, estimates the truncation error of the lower order method, $O\left(\Delta t^{2}\right)$ for $m=2$. The solution given by $\boldsymbol{y}^{2}$ (RK2) is accepted only if the error falls below some predefined tolerance $\epsilon_{\mathrm{tol}}$ (typically $10^{-5}$ ):

$$
\max \left[\left|\frac{p^{1}}{p^{2}}-1\right|, \max _{\kappa, i}\left(\left|X_{\kappa, i}^{1}-X_{\kappa, i}^{2}\right|\right)\right]<\epsilon_{\mathrm{tol}},
$$

where $(\kappa, i)$ extends to all ion species. A more accurate Runge-Kutta $(3,2)$ pair may be used instead. The condition (32) is usually satisfied in regions close to equilibrium ionization balance. If Eq. (32) is not fulfilled we switch to an explicit Runge-Kutta method of order 5 with an embedded 4th order solution with coefficients given by Cash-Karp, see Press et al. (1992), from now on CK45. The adaptive strategy provides a 5th-order accurate solution and allows us to split (if required) the full time step $\Delta t^{n}$ into a number of smaller sub-steps until the condition (32) is fulfilled in each one of them.

On the other hand, if Eq. (29) is not met, explicit time marching may potentially become unstable. In such situations, integration is carried using a 4th order semi-implicit Rosenbrock method with a 3rd order embedded error estimation (Ros34 henceforth). Rosenbrock schemes can be considered linearly implicit generalizations of Runge-Kutta methods, the prototype of which is the semi-implicit backward Euler method,

$$
\left(I-\Delta t^{n} J\right) \cdot\left(\boldsymbol{y}^{1}-\boldsymbol{y}^{0}\right)=\Delta t^{n} \boldsymbol{f}\left(\boldsymbol{y}^{0}\right) \text {. }
$$

These methods retain stability for large time steps at the additional cost of computing the Jacobian matrix $J=\partial \boldsymbol{f} / \partial \boldsymbol{y}$ of the system and performing matrix inversions by LU decomposition. Both features are notoriously time consuming for moderately large systems of equations, such as the one we deal with here. In Appendix B we show how the Jacobian can be computed using combined analytical and numerical differentiation. The full integration strategy is schematically illustrated in Fig. 3.

Alternatively, we also found satisfactory results by dividing the whole step $\Delta t^{n}$ into smaller ones and by sub-cycling with the explicit CK45 scheme. The sub-time stepping strategy has proved to handle the moderate stiffness arising at high $\left(T>10^{5}\right)$ temperatures, when the reaction rates become large. This makes, in our experience and for the tests presented in this work, the explicit scheme competitive with the semi-implicit method, inasmuch stiffness is spatially confined to a small fraction of the computational domain.

Once acceptable solutions $\boldsymbol{y}^{*}$ have been produced in every computational zone, we estimate the next time step according to the CFL stability restriction and the maximum fractional change produced during the radiation step:

$\Delta t^{n+2}=\min \left(\Delta t_{\mathrm{adv}}, \epsilon_{\mathrm{max}} \Delta t_{\mathrm{rad}}\right)$,

where, consistently with Eqs. (8) and (9), the minimum is taken over two consecutive time steps and $\Delta t_{\mathrm{adv}}$ is computed from the CFL condition. The quantity $\epsilon_{\max }$ specifies the maximum fractional change tolerance (typically $0.01 \lesssim \epsilon_{\max } \lesssim 0.1$ ) allowed during the source step. The radiative time step $\Delta t_{\text {rad }}$ is computed as

$\Delta t_{\mathrm{rad}}=\frac{\Delta t^{n}}{\max _{x y z}\left[\left|\frac{p^{0}}{p^{*}}-1\right|, \max _{\kappa, i}\left(\left|X_{\kappa, i}^{*}-X_{\kappa, i}^{0}\right|\right)\right]} \cdot$
Note that small values of $\epsilon_{\max }$ will result in a better coupling between the advection and source steps, at the cost of reducing the overall time step.

In terms of right-hand side evaluations, the computational overheads introduced by the selected algorithms (RK2:CK45:Ros34) are in the ratio 2:6:3 in each cell for a given time step. The semi-implicit method Ros 34 requires, however, 2 additional right-hand side calls to form the Jacobian (see Appendix B) and the inversion of a matrix by LU decomposition. This makes Ros34 the most computational expensive scheme of integration.

Nevertheless, extensive testing confirms that only a very small fraction of computational zones (usually $\lesssim 1 \%$ ) does actually require this special, but nonetheless crucial, treatment. The remaining vast majority of cells can be accurately evolved using a second order method. On the other hand, unconditional use of the CK45 or Ros34 algorithms throughout the whole grid leads to a noticeable loss of computational efficiency with no gain on the overall accuracy.

\subsection{Accuracy comparison}

In order to test the accuracy and efficiency of the selected time marching schemes adopted during the source step, we consider the evolution of a single parcel of gas departing from initial conditions far from equilibrium. This situation is typically encountered, for example, when a strong shock propagates into a cold medium. Neutral atoms crossing the front will suddenly feel the sharp increase in temperature and will try to readjust to the new conditions. The ionization timescale will be, most likely, much shorter than the typical advection scale. One is interested in performing the simulations at the timestep given by Eq. (34), but this can violate the condition expressed by Eq. (29).

We consider two cases in which a single computational zone is being evolved in time. Initial parameters have been found by running a full shock simulation like the one presented in Sect. 6.1, and selecting the computational zone showing the most extreme stiff conditions, according to Eq. (29). We perform a number of time integrations at constant step size using the Euler, RK2, CK45, and Ros34 algorithms previously described. Errors are computed with respect to a reference solution obtained with the CK45 integrator with a very stringent tolerance $\left(10^{-8}\right)$ and a small timestep $\left(\sim 10^{-4}\right.$ of the cooling timescale):

$\epsilon=\frac{\sum_{\kappa, i}\left|X_{\kappa, i}-X_{\kappa, i}^{\mathrm{ref}}\right|}{\sum_{\kappa, i} X_{\kappa, i}^{\mathrm{ref}}}$

The errors in temperature are lower in all cases because the equations of the chemical network can be, and usually are, more prone to very rapid variations (stiffness).

In the first case (top panel of Fig. 4), the initial temperature is set to $T=132000 \mathrm{~K}$, the initial neutral hydrogen fraction is $22 \%$ and the rest of the elements are in the highest ionization stage. Under these conditions, the ionization/recombination timescale is $\tau \approx 2 \times 10^{3} \mathrm{~s}$, typically much smaller than the scale on which hydrodynamical variables are transported, $\Delta t$.

At a fixed timestep $\Delta t=5 \tau$ (see middle panel in Fig. 4) RK2 is less accurate than Euler, this being a typical sign of stiffness (Ekeland et al. 1998). The integrator Ros34 yields the best accuracy, immediately followed by CK45. As the time step is further increased ( $\Delta t=50 \tau$, see bottom panel in Fig. 4), CK45 progressively loses accuracy, whereas Ros34 keeps the smallest errors. In this case, the use of a semi-implicit method clearly reveals its advantages. 


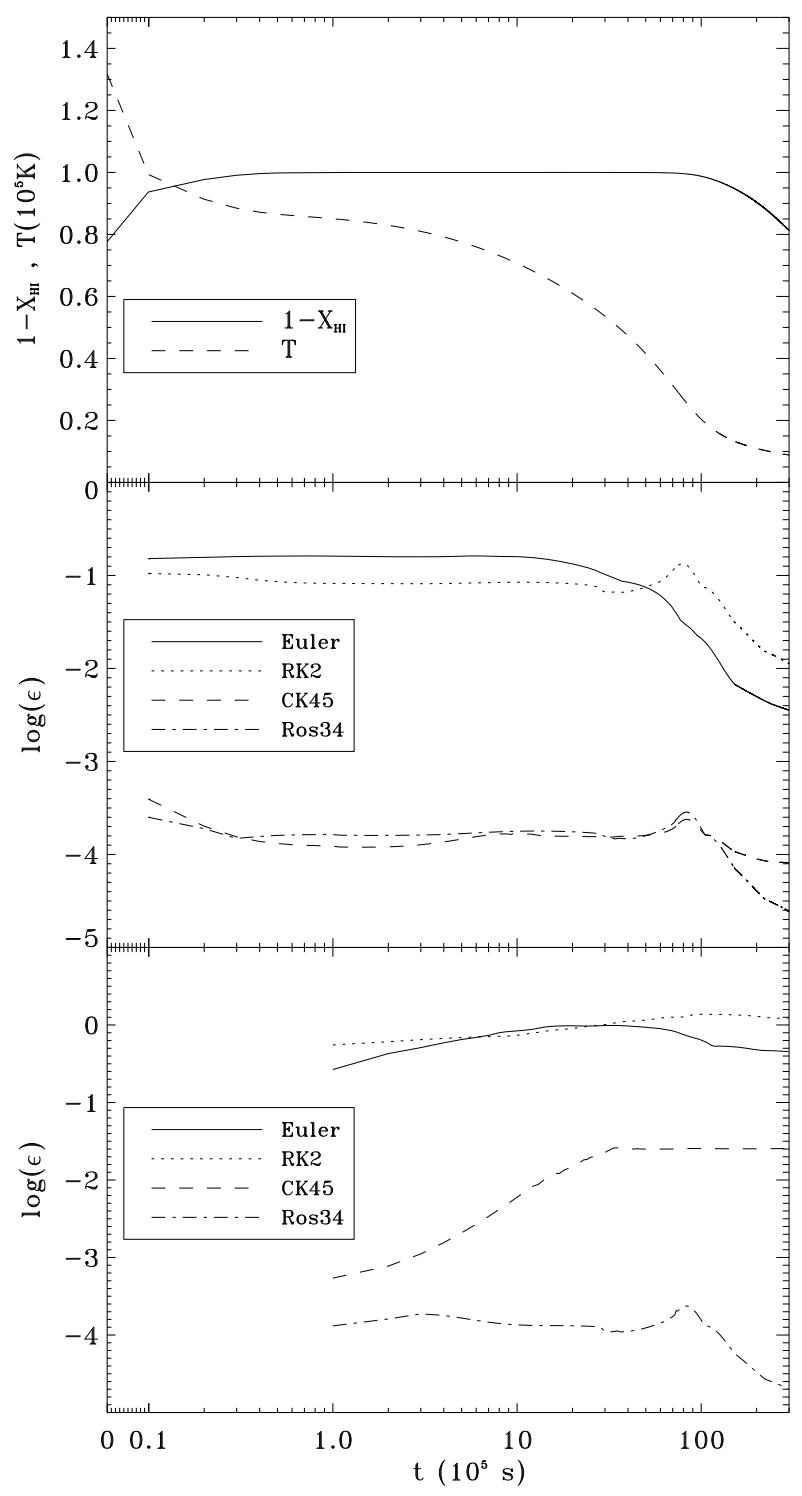

Fig. 4. Top panel: temperature and ionization fraction of hydrogen evolution; middle panel: relative errors in ionization fractions, for a fixed timestep $\Delta t=5 \tau$; bottom panel: same as middle, for a timestep $\Delta t=50 \tau$. The error plots begin at a time $\Delta t$ from the beginning of the simulation. Linear time axis up to $10^{4} \mathrm{~s}$, logarithmic after.

In the second test, we consider a fully-ionized gas (except for hydrogen, $X_{\mathrm{HI}} \approx 69 \%$ ) at low temperature $T=10^{4} \mathrm{~K}$. For this choice of parameters, the recombination timescale is even smaller than before, $\tau \approx 10^{3}$ s. Figure 5 shows the errors computed with selected integration algorithms when $\Delta t=100 \tau$. The resulting accuracies confirm the trend found for the previous case: low-order, non-adaptive time marching schemes are not suitable in conditions far from equilibrium. On the contrary, Ros34 being an adaptive semi-implicit scheme, does not suffer from this loss in accuracy and turns out to be the best integration method. Explicit adaptive algorithms such as CK45, still exhibits somewhat better results than the lower order methods. It should also be mentioned that the accuracy of explicit schemes may be further improved if step sub-cycling is used.

We conclude that, for the slow varying regions of the MHD simulation, RK2 (or RK3) can be a good choice, while for very fast varying regions a higher-order integrator with time step adaptivity (as CK45) or even an implicit one (Ros34) become

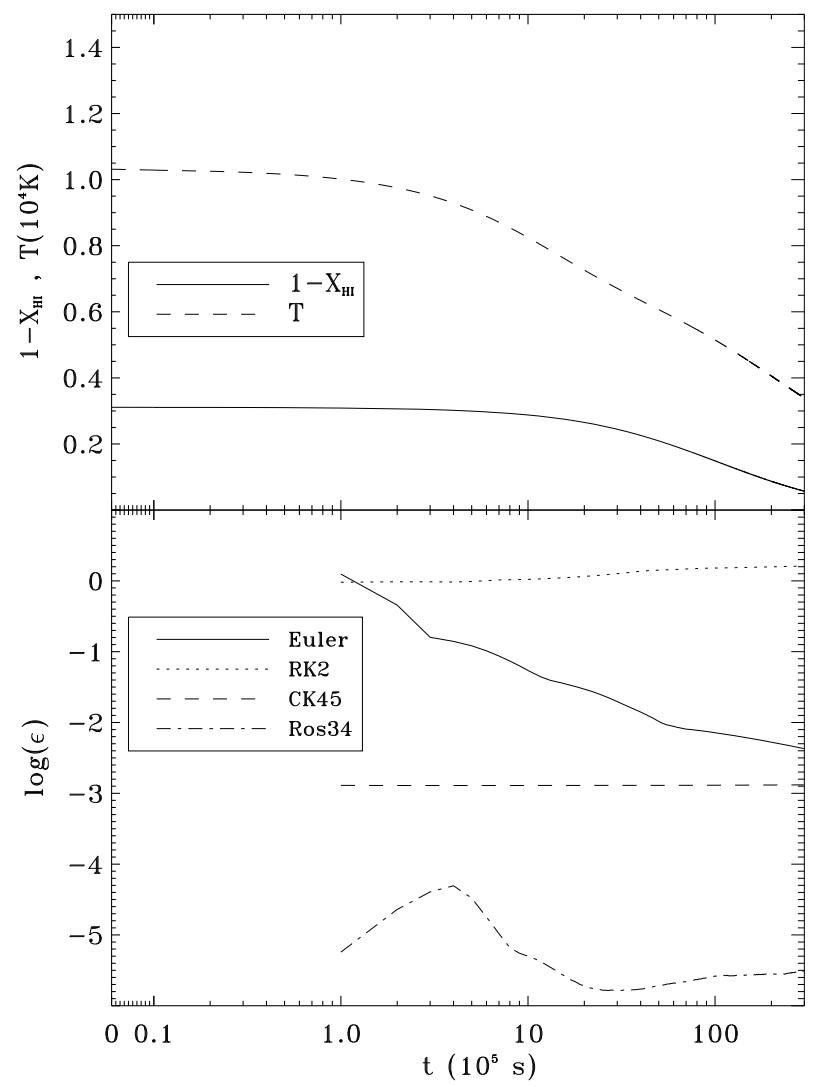

Fig. 5. Top panel: temperature and ionization fraction of hydrogen evolution; bottom panel: relative errors in ionization fractions, for a fixed timestep $\Delta t=100 \tau$. Linear time axis up to $10^{4} \mathrm{~s}$, logarithmic after.

necessary. Also, large temperatures are not a necessary condition for the system of equations to become stiff, since this can also happen at relatively low temperatures when the ionization/recombination rates are high.

\section{Astrophysical applications}

We now apply the newly-developed cooling function to problems of astrophysical interest. First, we consider a single, onedimensional radiative shock propagating in a stratified medium with decreasing density. Then, an example of application of the first setup for the computation of emission line ratios is shown. Finally, a study of the dynamical evolution of a jet with varying ejection velocity in two-dimensional axial symmetry is presented.

\subsection{Propagating shocks}

It is interesting to see the difference radiative losses make in the dynamical evolution of a propagating shock. A first series of tests were made in 1D, with an initial perturbation in pressure, density, and velocity that propagates in a stratified medium of $T_{\text {pre }}=1000 \mathrm{~K}$ and becomes a shock. The pre-shock density in the external medium is

$\rho_{0}(x)=\rho_{0} \frac{x_{0}^{2}}{x_{0}^{2}+x^{2}}$,

where $x$ is the spatial coordinate and the departure density $\rho_{0}$ corresponds to a particle number density $N_{0}=10^{5} \mathrm{~cm}^{-3}$. This 


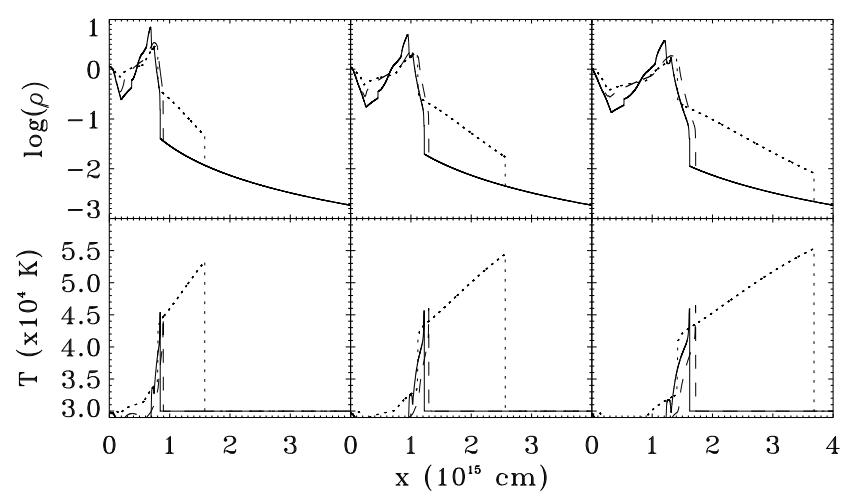

Fig. 6. Density logarithmic profiles (top row) and temperature profiles (bottom row), at three evolutionary stages: adiabatic (dotted line), with SNEq (dashed line), and MINEq (solid line) cooling.

density distribution should approximate well the density in an expanding jet. The initial perturbation is set in such a way that only one shock forms instead of the usual pair of forward/reverse shocks. The setup is described in detail in Massaglia et al. (2005). The 1D simulation was run on a domain of length $L=4 \times 10^{15} \mathrm{~cm}$, with a resolution of $1.4 \times 10^{11} \mathrm{~cm}$, and the initial velocity perturbation had an amplitude $\Delta v=30 \mathrm{~km} \mathrm{~s}^{-1}$.

In Fig. 6, a comparison is made between the evolution of the formed shock in the absence of cooling, with SNEq and the evolution with MINEq. Plots of density and temperature are presented at three evolutionary instants in the propagation. As it results from the plots in Fig. 6, the shock dynamics are heavily influenced by radiative cooling. The shock propagation velocity decreases almost twice in the simulations with cooling with respect to the adiabatic ones. The smooth decrease in temperature after the shock front in the adiabatic simulation is replaced by a much sharper one in the simulations with cooling.

The differences between the density plots obtained with the two cooling models are quantitatively moderate, while the shapes are similar. The maximum temperatures attained are very close in the two cooling models. Overall, the dynamical differences that appear between the use of the two cooling models are small, but the line intensity ratios are very sensitive to density/temperature conditions so the differences may result in moderate amplitudes.

The test in equivalent configuration was also performed in a 2D slab. The results were, as expected, very similar to the ones from the 1D simulations, with somewhat smoother curves due to the lower resolution employed. It results that for simulations of propagating shocks like the one described it is very important to include the radiative cooling losses, which heavily influence the dynamics. A simplified treatment of these losses can be sufficient for studies on the dynamics, while for the computation of emission line maps the detailed (MINEq) approach is more suitable. An example is presented in the following section.

\subsection{Emission lines}

The computation of emission line ratios from numerical simulations is of great importance for the field to compare to observations and to discriminate between theoretical astrophysical models.

In a simple 1D setup, one of the ways to model a YSO jet and to estimate the emission is the following. Supposing that the emission comes from shocks inside the jet, the propagation of

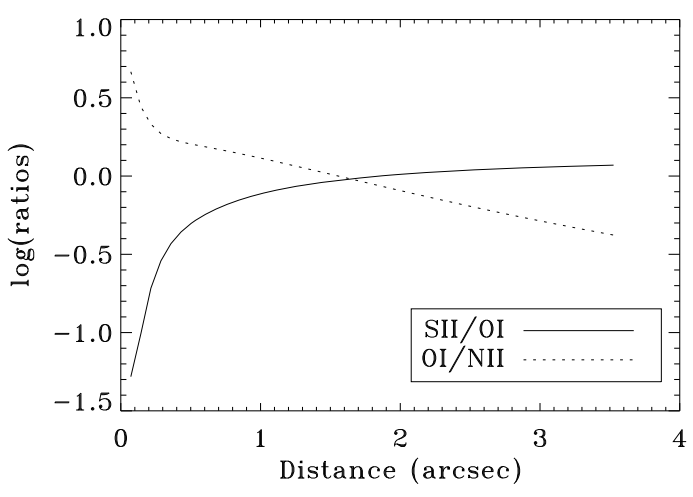

Fig. 7. Line ratios with MINEq cooling, for the propagating shock described in the previous section.

a shock resulting from a velocity fluctuation is simulated in the frame of reference of the jet material. The emission in the chosen lines is computed and averaged over a space region corresponding to the resolution of the observational data (in our example $10^{15} \mathrm{~cm}$ ) for each evolutionary step. Then, the resulting averaged line ratios are plotted at space points corresponding to the transport speed of the jet material, set to $150 \mathrm{~km} \mathrm{~s}^{-1}$.

The computation was done for the setup presented in Sect. 6.1. The resulting plot, presented in Fig. 7, has also the $x$ axis converted in arcseconds (for a distance $D=140 \mathrm{pc}$ ) and represents the emission of a jet in the assumption that the emission comes from internal shocks formed due to initial jet velocity variability. We present ratios between the forbidden emission lines of O I $\lambda \lambda 6300 \AA+6364 \AA$, N II $\lambda \lambda 6548 \AA+6583 \AA$ and S II $\lambda \lambda 6716 \AA+6731 \AA$. Such synthetic emission line ratios can be directly compared with observations of YSO jets.

The main advantage in using MINEq for creating synthetic emission maps is that non-equilibrium ionization balance for the elements are provided. The computation of the emission in selected lines is done in post-processing, with routines distributed together with the code.

\subsection{Jet propagation}

Observations of YSO jets that show series of emission knots along their length pointed out that simple steady-state models cannot explain their morphology. These knots have been interpreted in the literature as due either to the nonlinear evolution of Kelvin-Helmholtz instabilities set at the jet-ambient interface (Micono et al. 2000) or to velocity variabilities of the beam (Internal Working Surfaces, see for example Raga et al. 2002) that, during their propagation, steepen into shocks. The latter scenario has been chosen as a possible astrophysical application of the cooling module.

In the present case, we consider a variable jet in $2 \mathrm{D}$ cylindrical geometry propagating into a uniform ambient medium with particle number density $n_{\mathrm{a}}=200 \mathrm{~cm}^{-3}$ and temperature $T_{\mathrm{a}}=2500 \mathrm{~K}$. The beam is injected at the $z=0, r<R_{\mathrm{j}}$ $\left(R_{\mathrm{j}}=2.5 \times 10^{15} \mathrm{~cm}\right)$ boundary with higher density $\left(n_{\mathrm{j}}=5 n_{\mathrm{a}}\right)$ than the background. The mean jet velocity is $v_{\mathrm{j}}=110 \mathrm{~km} \mathrm{~s}^{-1}$ with sinusoidal oscillations of amplitude $\Delta v=25 \mathrm{~km} \mathrm{~s}^{-1}$ and a period of $\tau=50 \mathrm{yrs}$. 


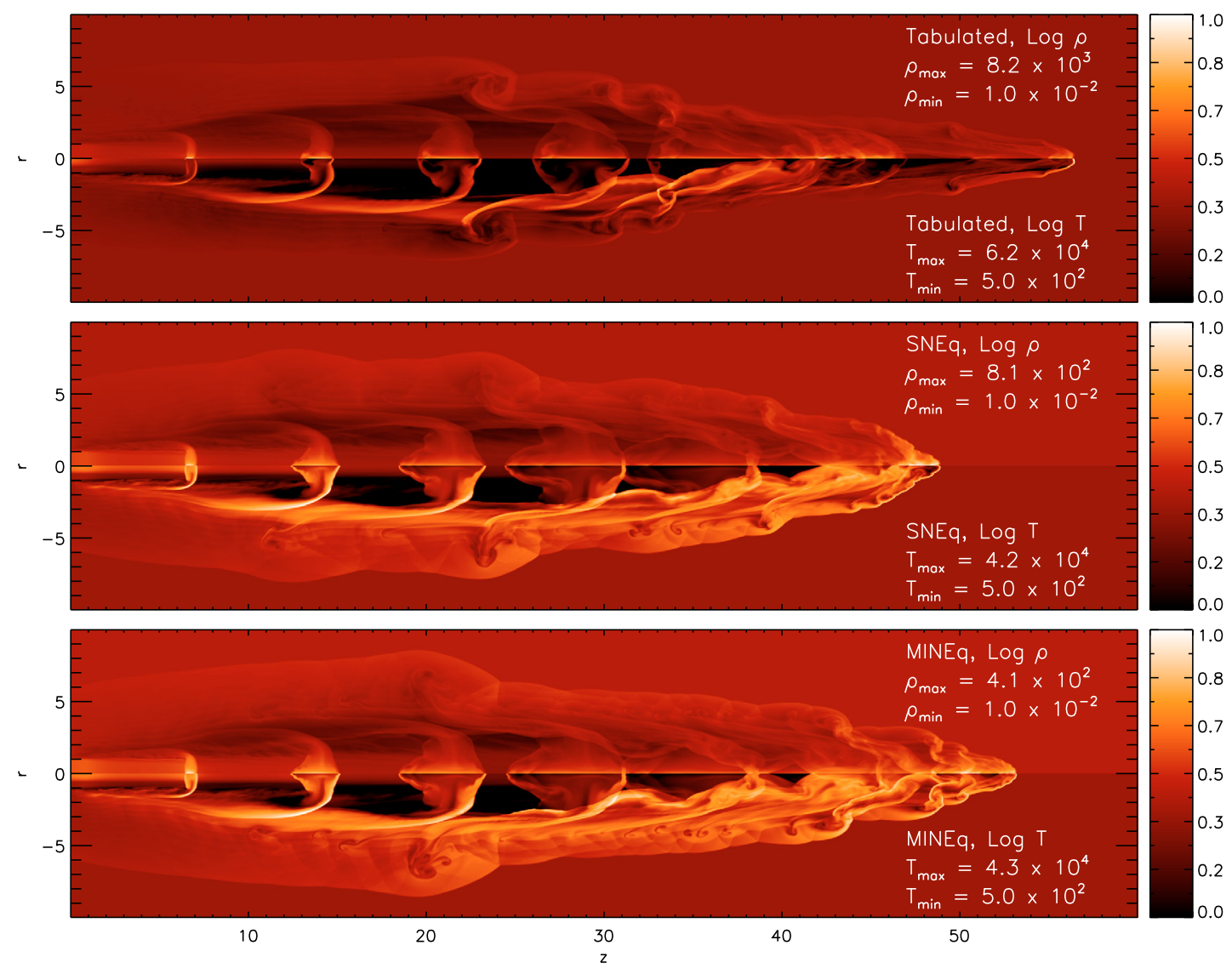

Fig. 8. Tabulated Cloudy (top panel), radiative with SNEq cooling (middle panel) and MINEq cooling (bottom panel) for the jet simulation. In each panel, the upper and lower halves show density and temperature in log scale, respectively. The different shades are normalized between $\rho_{\text {min }}$ and $\rho_{\max }$ (for density), $T_{\min }$ and $T_{\max }$ (for temperature).

A purely toroidal magnetic field is injected at $z=0$ along with the beam, following the simple configuration described in Lind et al. (1989):

$B_{\phi}(r)= \begin{cases}B_{m} \frac{r}{a} & \text { for } 0 \leq r<a, \\ B_{m} \frac{a}{r} & \text { for } a \leq r<R_{\mathrm{j}}, \\ 0 & \text { otherwise, }\end{cases}$

where $B_{m}$ and $a$ are the magnetization strength and radius. Demanding pressure equilibrium at the jet inlet, $\mathrm{d}\left(p+B_{\phi}^{2}\right) / \mathrm{d} r=$ $-B_{\phi}^{2} / r$, one recovers the pressure profile inside the beam $\left(r \leq R_{\mathrm{j}}\right)$,

$p(r)=p_{0}-B_{m}^{2} \min \left(1, \frac{r^{2}}{a^{2}}\right)$

where $p_{0}$ is corresponds to a central temperature $T_{0}=10000 \mathrm{~K}$. Finally, the magnetization strength, $B_{m}$ is prescribed from the plasma $\beta$ parameter, defined in terms of the averaged beam pressure:

$\beta \equiv \frac{2}{B_{m}^{2}} \frac{\int_{0}^{R_{\mathrm{j}}} p(r) r \mathrm{~d} r}{\int_{0}^{R_{\mathrm{j}}} r \mathrm{~d} r}=\frac{a^{2}}{R_{\mathrm{j}}^{2}}+2 \frac{p_{0}}{B_{m}^{2}}-2$

from which one can easily recover $B_{m}$. For the present computation, we set $a=0.6 R_{\mathrm{j}}$ and $\beta=1$. This choice of parameters is similar to the ones found by Masciardi \& Raga (2001) in their attempt to model the curved $\mathrm{HH} 505$ jet.
Numerical integration is carried out with the PPM method and the HLLC Riemann solver of Li (2005). We use 30 zones on the jet radius and the domain extends, in beam radii, from 0 to 10 in the radial direction and from 0 to 60 in the longitudinal direction. Free outflow is assumed across the outer boundaries, whereas reflecting boundary conditions hold at the axis $(R=0)$ and outside the jet inlet $\left(z=0, r>R_{\mathrm{j}}\right)$. A smoothing function is introduced for all variables at the transition between the jet material and the external medium to avoid the formation of an unphysical high temperature low-density layer around the jet.

We perform a set of three simulations, by adopting i) a tabulated cooling function; ii) simplified treatment of radiative cooling losses (SNEq); and iii) the detailed cooling treatment. The tabulated cooling function simply consists in adding a source term to the energy equation, given by the Cloudy $Z=1$ cooling curve (presented in Sect. 4.3) as a tabulated function of temperature. This cooling implementation does not follow the ionization balance of any element, but as a standard procedure for this kind of approach, the effective cooling function is multiplied by the particle density squared to obtain energy losses per unit volume. Results at $t \approx 500$ yrs are shown in Fig. 8 .

The pulsed initial jet velocity produces, as expected, a number of intermediate shocks propagating along the jet with typical post-shock temperatures in the range $15000 \div 25000 \mathrm{~K}$. The morphology is similar between the SNEq and MINEq runs, since at these temperatures the two cooling losses are comparable. Larger deviations are observed close to the head of the jet, where temperatures are higher and the two cooling functions exhibit larger differences. Overall, while SNEq and MINEq 


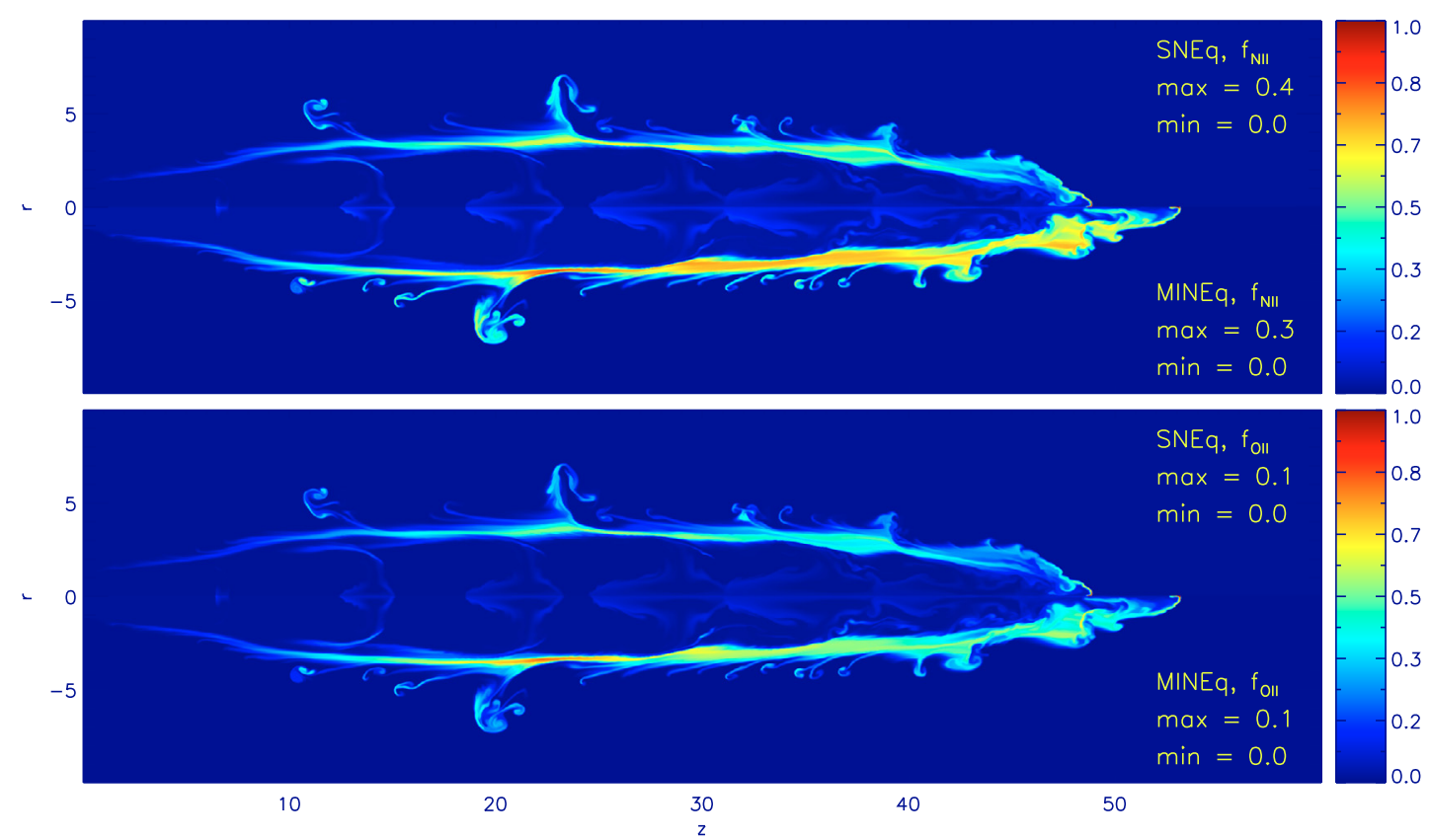

Fig. 9. Fractions of N II (top panel) and O II (bottom panel) for the jet simulation. In each panel, the upper and lower halves show the results with SNEq and MINEq, respectively. The different shades are normalized between the minimum and maximum values of the fractions.

give similar results, radiated losses are higher for the tabulated Cloudy cooling, as can be inferred by the reduced lateral expansion of the cocoon (this can be expected considering the effective cooling curves in Fig. 2).

Nevertheless, temperatures at the jet head are highest for the tabulated cooling case. In order to understand this apparently unexpected result, we have performed a systematic comparison between the Tabulated and SNEq cooling functions by means of supplementary simulations (not shown here). Our results demonstrate that at high temperatures $\left(\gtrsim 4 \times 10^{4} \mathrm{~K}\right)$ and low ionization, the SNEq line emission becomes larger than the equilibrium Cloudy one. In this case, it is crucial that the SNEq line emissions depend on the electron number density and that this density is dynamically computed from the non-equilibrium ionization of $\mathrm{H}$. In the tabulated case, an equilibrium ionization balance is implicitly assumed. This confirms that the maximum temperatures can be an indication of the maximum cooling losses attained locally, but not on the overall cooling losses. It must also be noticed that the resolution of these simulations is still low to resolve the post-shock zone at the jet head, so the maximum temperatures observed may be subject to large uncertainties.

We conclude that even a simple cooling like SNEq, evolving only the hydrogen fraction, is a much better approximation than using tabulated cooling losses.

The ionization fractions computation is very important when it comes to producing synthetic emission maps in various emission lines to be compared with observations. In Fig. 9, the fractions of N II and O II are presented, dynamically computed by MINEq and alternatively computed from SNEq considering them fixed by the hydrogen ionization through charge-transfer (see Osterbrock \& Ferland 2005). The differences are moderate and can result in variations of $20-30 \%$ in the emission lines computation.

The steep gradients and transition regions forming immediately behind the shock front are crucial in determining the emission properties, e.g. line intensity ratios. For this reason they need to be accurately resolved (Massaglia et al. 2005). However, at the resolution employed here $(300 \times 1800)$, only the general physical evolution can be captured. Considerably higher resolution is required and this can be efficiently achieved only through adaptive mesh refinement simulations. This issue will be the subject of forthcoming works.

\section{Conclusions}

After a series of tests and validation, the detailed treatment of radiative losses MINEq is now implemented in the MHD code PLUTO. The choice of the integration technique and the optimizations are, as far as we know, unique at the time of this writing and provide a high degree of accuracy in ion species abundances and radiative losses computation. Both theoretical and technical aspects of the current implementation, as well as testing process and applications were discussed in the previous sections.

A major advantage obtained by using MINEq, compared to previously employed SNEq, is that the line emission can be computed in conditions of non-equilibrium ionization for all species, more likely to be encountered in situations of rapid changes, as it is the case of shock waves.

As shown by the tests presented, the choice of the cooling model between MINEq and SNEq, has an increasing effect on the structure formation whenever temperatures exceed $25000 \mathrm{~K}$, and has an important influence for the ionization fractions that reflects in the emission line computations. For a preliminary dynamical study, a tabulated cooling function that does not integrate any ion specie can be employed, but the relatively low computational cost of a cooling that evolves the hydrogen ionization in a time-dependent fashion makes the latter advisable in most cases. Whenever the purpose is the computation of emission line ratios, employing a detailed cooling like MINEq produces more reliable results as the ionization fractions are followed in nonequilibrium conditions, which are likely to be encountered in the real astrophysical objects.

An important feature of the cooling model implementation is that it is upgradeable, more ion species and other processes can be added (increasing also the temperature range of application). 
Also, it can be used as starting point for the integration of atomic chemistry and cooling processes to other MHD codes.

The newly-developed cooling function provides a powerful tool for investigating the stellar jets and gaseous nebulae. It is, in the current configuration, suited for the study of radiative shocks in stellar jets. High resolution, adaptive numerical simulations to predict line emission in YSO jets will be subject of forthcoming works.

\section{Appendix A: Ionization/recombination processes}

The following processes are taken into consideration: collisional ionization, radiative, and dielectronic recombination, chargetransfer with $\mathrm{H}$ and $\mathrm{He}$. These processes enter the ionization/recombination coefficients defined and used in Sect. 3.

\section{A.1. Collisional ionization}

We use the Voronov (1997) data to estimate the collisional ionization rates with the analytical formula:

$\zeta^{\text {coll }}=A \cdot \frac{1+P \cdot U^{1 / 2}}{X+U} \cdot U^{K} \cdot \mathrm{e}^{-\mathrm{U}}$,

where $U=\Delta E / T$ and $A, P, \Delta E, X$, and $K$ parameters are listed in Table 1 of the cited paper. $T$ and $\Delta E$ are expressed in $\mathrm{eV}$, and $\zeta^{\text {coll }}$ in $\mathrm{cm}^{3} \mathrm{~s}^{-1}$.

The actual number of ionizations in unit time and unit volume will be:

$\frac{\mathrm{d} N}{\mathrm{~d} t}=N_{i} \cdot N_{\mathrm{el}} \cdot \zeta^{\text {coll }}$

where $N_{i}$ is the total number density of atoms in the lowerionization state, and $N_{\mathrm{el}}$ the electron number density.

\section{A.2. Radiative recombination}

The total radiative recombination rates are taken from Pèquignot et al. (1991). The total recombination rate is fitted with the analytical formula:

$\alpha^{\mathrm{RR}}=10^{-13} z \frac{a t^{b}}{1+c t^{d}}$,

where $z$ is the ionic charge and $t=10^{-4} T(K) / z^{2}$. The four parameters $a, b, c$, and $d$ are given in Table 1 in the cited paper.

The resulting $\alpha^{\mathrm{RR}}$ is expressed in units of $\mathrm{cm}^{3} \mathrm{~s}^{-1}$.

\section{A.3. Dielectronic recombination}

The dielectronic recombination process proceeds as

$A_{p}^{+\mathrm{m}+1}+\mathrm{e}^{-} \rightarrow A_{a}^{+\mathrm{m}} \rightarrow A_{b}^{+\mathrm{m}}+h v$,

where $p$ stands for a state of the $m+1$ times ionized element $A$, and $a$ and $b$ represent an auto-ionizing and a true bound state of the next ionization stage.

From Nussbaumer \& Storey (1983), the dielectronic recombination rates are fitted by the analytical formula:

$\alpha^{\mathrm{DR}}=10^{-12}\left(\frac{a}{t}+b+c t+c t^{2}\right) t^{-3 / 2} \exp \left(\frac{-f}{t}\right)$,

where $t=T(K) / 10^{4} \mathrm{~K}$ and $\alpha^{\mathrm{DR}}$ is expressed in $\mathrm{cm}^{3} \mathrm{~s}^{-1}$. The coefficients are given in a table from the cited paper. We used the data from Nussbaumer \& Storey (1983) for the C, N, and $\mathrm{O}$ ions.

\section{A.4. Total electron - ion recombination}

For the He, Ne, and S ions, data from Kato \& Asano (1999) was used for the total recombination coefficient (radiative + dielectronic). These are tabulated values that we interpolate in our temperature range.

\section{A.5. Charge transfer with $H$}

The charge transfer (exchange) reactions with $\mathrm{H}$ are reactions of the form:

$A^{+n}+\mathrm{H} \rightleftarrows A^{+(n-1)}+\mathrm{H}^{+}+\delta E$.

The direct reaction is called charge-transfer recombination and the inverse charge-transfer ionization $\left(\zeta^{\mathrm{HII}}\right)$.

We took the data for charge transfer with $\mathrm{H}$ from Kingdon \& Ferland (1996). The recombination/ionization rate is fitted by the analytical formula

$\alpha^{\mathrm{HI}}, \zeta^{\mathrm{HII}}=a t_{4}^{b}\left[1+c \mathrm{e}^{\mathrm{d} t_{4}}\right]$,

where $t_{4}=T(\mathrm{~K}) / 10^{4} \mathrm{~K}$ and the parameters $a, b, c$, and $d$ are listed in Tables 1 and 3 from the cited paper.

\section{A.6. Charge transfer with $\mathrm{He}$}

The charge transfer (exchange) reactions with He are reactions of the form:

$A^{+\mathrm{n}}+\mathrm{He} \rightarrow A^{+(\mathrm{n}-1)}+\mathrm{He}^{+}$.

We took the data for charge transfer with He from Wang et al. (2001) and references herein. The recombination rate is fitted by the analytical formula

$\alpha^{\mathrm{HeI}}=a t_{4}^{b}\left[1+c \exp \left(\mathrm{d} t_{4}\right)\right]$

where $t_{4}=T / 10^{4} \mathrm{~K}$ and the parameters $a, b, c$, and $d$ are listed in tables available on-line.

\section{Appendix B: Jacobian matrix}

The solution of implicitly linearized equations such as Eq. (33) or the Rosenbrock scheme requires the expression of the Jacobian matrix of the system of equations given by (10). Using the definition,

$J \equiv \frac{\partial \boldsymbol{f}(\boldsymbol{y})}{\partial \boldsymbol{y}}=\left(\begin{array}{ll}\frac{\partial \dot{\boldsymbol{X}}}{\partial \boldsymbol{X}} & \frac{\partial \dot{\boldsymbol{X}}}{\partial p} \\ \frac{\partial \dot{p}}{\partial \boldsymbol{X}} & \frac{\partial \dot{p}}{\partial p}\end{array}\right)$

where $\boldsymbol{y}=\{p, \boldsymbol{X}\}$ and $\boldsymbol{f}(\boldsymbol{y}) \equiv \dot{\boldsymbol{y}}$. Partial derivatives are computed using combined analytical and numerical differentiation. We note in the first place that, for practical reasons, the righthand side $\boldsymbol{f}(p, \boldsymbol{X})$ is better expressed in terms of temperature and ionization fractions, that is

$f(p, X) \equiv g(T, X)$

where $T, p$, and $\boldsymbol{X}$ are related through

$T=\frac{p}{\rho} \frac{m_{\mathrm{u}} \mu(\boldsymbol{X})}{k_{\mathrm{B}}}$ 
This allows us to compute partial derivatives with respect to the ion fractions using the chain rule,

$$
\left.\frac{\partial \boldsymbol{f}}{\partial X_{\xi, m}}\right|_{p}=\left.\frac{\partial \boldsymbol{g}}{\partial X_{\xi, m}}\right|_{T}+\left.\frac{\partial \boldsymbol{g}}{\partial T}\right|_{\boldsymbol{X}} \frac{\partial T}{\partial X_{\xi, m}}
$$

where, using Eq. (B.3) and the definitions of the mean molecular weight, Eq. (12), we can express the second term on the right as

$$
\left.\frac{\partial \boldsymbol{g}}{\partial T}\right|_{\boldsymbol{X}} \frac{\partial T}{\partial X_{\xi, m}}=\left.\frac{\partial \boldsymbol{f}}{\partial p}\right|_{\boldsymbol{X}} \frac{p}{\mu}\left[\frac{1}{\mu_{\mathrm{D}}} \frac{\partial \mu_{\mathrm{N}}}{\partial X_{\xi, m}}-\frac{\mu}{\mu_{\mathrm{D}}} \frac{\partial \mu_{\mathrm{D}}}{\partial X_{\xi, m}}\right],
$$

where the term is square brackets is simply $\partial \mu(\boldsymbol{X}) / \partial X_{\xi, m}$ whereas $\mu_{\mathrm{N}}$ and $\mu_{\mathrm{D}}$ are, respectively, the numerator and the denominator of the mean molecular weight.

The explicit dependence on $T$ and $\boldsymbol{X}$ in our ionization network, Eq. (21), is made clear by rearranging terms as

$$
\begin{aligned}
\dot{X}_{\kappa, i}= & L_{\kappa, i}(T, X) X_{\kappa, i-1}-C_{\kappa, i}(T, X) X_{\kappa, i} \\
& +R_{\kappa, i}(T, X) X_{\kappa, i+1},
\end{aligned}
$$

for each element's ions. The coefficients $L_{\kappa, i}, C_{\kappa, i}$, and $R_{\kappa, i}$ are expressed by sums of functions depending on either $T$ or $\boldsymbol{X}$ :

$$
\begin{aligned}
& L_{\kappa, i}=L_{\kappa, i}^{a} N_{\mathrm{el}}+L_{\kappa, i}^{b} X_{\mathrm{HI}}+L_{\kappa, i}^{c} X_{\mathrm{HeI}}+L_{\kappa, i}^{d} \\
& C_{\kappa, i}=C_{\kappa, i}^{a} N_{\mathrm{el}}+C_{\kappa, i}^{b} X_{\mathrm{HI}}+C_{\kappa, i}^{c} X_{\mathrm{HeI}}+C_{\kappa, i}^{d} \\
& R_{\kappa, i}=R_{\kappa, i}^{a} N_{\mathrm{el}}+R_{\kappa, i}^{b} X_{\mathrm{HI}}+R_{\kappa, i}^{c} X_{\mathrm{HeI}}+R_{\kappa, i}^{d}
\end{aligned}
$$

where the $L_{\kappa, i}^{\cdots}$ 's, $C_{\kappa, i}^{\cdots}$ 's, and $R_{\kappa, i}^{\cdots}$ 's depend on $T$ only whereas $N_{\mathrm{el}}$, given by Eq. (15), depends on $\boldsymbol{X}$ only. Focusing on the Jacobian sub-matrix $\partial \dot{\boldsymbol{X}} / \partial \boldsymbol{X}$, we evaluate the first term in Eq. (B.4) as

$$
\begin{aligned}
\left.\frac{\partial \dot{X}_{\kappa, i}}{\partial X_{\xi, m}}\right|_{T}= & L_{\kappa, i} \delta_{i-1, m} \delta_{\kappa, \xi}+\frac{\partial L_{\kappa, i}}{\partial X_{\xi, m}} X_{\kappa, i-1}-C_{\kappa, i} \delta_{i, m} \delta_{\kappa, \xi}-\frac{\partial C_{\kappa, i}}{\partial X_{\xi, m}} X_{\kappa, i} \\
& +R_{\kappa, i} \delta_{i+1, m} \delta_{\kappa, \xi}+\frac{\partial R_{\kappa, i}}{\partial X_{\xi, m} X_{\kappa, i+1}}
\end{aligned}
$$

where $\delta_{i, m}$ is the Kronecker delta symbol and

$$
\begin{aligned}
\frac{\partial L_{\kappa, i}}{\partial X_{\xi, m}}= & L_{\kappa, i}^{a}(T) N \gamma_{m} A_{\xi}+L_{\kappa, i}^{b}(T) \delta_{\mathrm{HI}, \xi m} \\
& +L_{\kappa, i}^{c}(T) \delta_{\mathrm{HeI}, \xi m} \\
\frac{\partial C_{\kappa, i}}{\partial X_{\xi, m}}= & C_{\kappa, i}^{a}(T) N \gamma_{m} A_{\xi}+C_{\kappa, i}^{b}(T) \delta_{\mathrm{HI}, \xi m} \\
& +C_{\kappa, i}^{c}(T) \delta_{\mathrm{HeI}, \xi m} \\
\frac{\partial R_{\kappa, i}}{\partial X_{\xi, m}}= & R_{\kappa, i}^{a}(T) N \gamma_{m} A_{\xi}+R_{\kappa, i}^{b}(T) \delta_{\mathrm{HI}, \xi m} \\
& +R_{\kappa, i}^{c}(T) \delta_{\mathrm{HeI}, \xi m} .
\end{aligned}
$$

In the previous equations we made use of the fact that $\partial N_{\mathrm{el}} / \partial X_{\kappa, i}=N\left(\gamma_{i}-1\right) B_{\kappa}$.

The last row of the Jacobian involves derivatives of the cooling function with respect to $\boldsymbol{X}$ :

$$
\frac{\partial \dot{p}}{\partial X_{\xi, m}}=-N_{\mathrm{at}} \gamma_{m} B_{\xi} \frac{\Lambda}{N_{\mathrm{el}}}-N_{\mathrm{at}} N_{\mathrm{el}} \frac{\partial \Lambda}{\partial X_{\xi, m}}-\frac{\partial L_{\mathrm{FF}}}{\partial X_{\xi, m}}-\frac{\partial L_{\mathrm{I}-\mathrm{R}}}{\partial X_{\xi, m}}
$$

where

$$
\frac{\partial \Lambda}{\partial X_{\xi, m}}=\mathcal{L}_{\xi, m} B_{\xi}+N \gamma_{m} B_{\xi} \sum_{\kappa, i} X_{\kappa, i} \frac{\partial \mathcal{L}_{\kappa, i}}{\partial X_{\xi, m}} B_{\beta},
$$

$\partial \mathcal{L}_{\kappa, i} / \partial X_{\xi, m}$ is found numerically and the remaining terms are found by straightforward differentiation of the energy losses due to ionization-recombination and bremsstrahlung.

Finally, partial derivatives with respect to pressure needed in Eq. (B.5) and in the last column of $J$ are computed numerically using a centered approximation:

$$
\frac{\partial f}{\partial p} \approx \frac{f(p(1+\epsilon), \boldsymbol{X})-f(p(1-\epsilon), \boldsymbol{X})}{\epsilon p}
$$

where $\epsilon$ is a small parameter (typically $\epsilon=10^{-4}$ ).

Acknowledgements. This work has been supported by the EU contract MRTNCT-2004-005592 within the Marie Curie RTN JETSET. The Cloudy curves were created by Michal Rozyczka and Guillermo Tenorio-Tagle and later updated by Tomek Plewa with the help of version 90.01. O.T. wishes to thank Dr. Tomek Plewa for comments on the Cloudy results. We thank the referee for valuable comments and observations useful in improving the paper.

\section{References}

Bacciotti, F. 2004, ApJS, 293, 37

Blondin, J. M., \& Lufkin, E. A. 1993, ApJS, 88, 589

Blum, R. D., \& Pradhan, A. K. 1992, ApJS, 80, 425

Berger, M. J., \& LeVeque, R. J. 1998, SIAM J. Num. Anal., 35, 6, 2298

Dalgarno, A., \& McCray, R. A. 1972, ARA\&A, 10, 375

Ekeland, K., Owren, B., \& Øines, E. 1998, ACM Trans. Math. Softw., 24, 368

Ferland, G. J., Korista, K. T., Verner, D. A., et al. 1998, PASP, 110, 761

Giovanardi, C., Natta, A., \& Palla, F. 1987, A\&AS, 70, 269

Kato, T., \& Asano, E. 1999, National Institute for Fusion Science - Japan, NIFSDATA-54

Kingdon, J. B., \& Ferland, G. J. 1996, ApJS, 106, 205

Lavalley-Fouquet, C., Cabrit, S., \& Dougados, C. 2000, A\&A, 356, L41

Leahy, J. P., \& Williams, A. G. 1984, MNRAS, 210, 929

Li, Shengtai 2005, J. Comput. Phys., 203, 344

Lind, H., Payne, D., Meier, D., \& Blandford, R. 1989, ApJ, 344, 89

Marten, H., \& Szczerba, R. 1997, A\&A, 325, 1132

Masciadri, E., \& Raga, A. C. 2001, AJ, 121, 408

Massaglia, S., Mignone, A., \& Bodo, G. 2005, A\&A, 442, 549

Micono, M., Bodo, G., Massaglia, S., et al. 2000, A\&A, 360, 795

Mignone, A., Massaglia, S., Bodo, G., et al. 2007, ApJS, 170, 228

Nussbaumer, H., \& Storey, P. J. 1983, A\&A, 125, 75

Osterbrock, D. E., \& Ferland, G. J. 2005, Astrophysics of Gaseous Nebulae and Active Galactic Nuclei (University Science Books)

Pèquignot, D., Petitjean, P., \& Boisson, C. 1991, A\&A, 251, 680

Poludnenko, A., Varnière, P., Cunningham, A., Frank, A., \& Mitran, S. 2005, Lecture Notes in Computational Science and Engineering (Springer), 41, 331

Pradhan, A. K., \& Zhang, H. L. 1999, Landolt-Boernstein Volume 17: Photon and Electron Interactions with Atoms, Molecules, Ions (Springer-Verlag), I.17.B, 1

Press, W. H., Flannery, B. P., Teukolsky, S. A., \& Vetterling, W. T. 1992, Numerical Recipes in C (Cambridge University Press)

Raga, A. C., Mellema, G., \& Lundqvist, P. 1997, ApJS, 109, 517

Raga, A. C., Navarro-Gonzàlez, R., \& Villagràn-Muniz, M. 2000, Rev. Mex. Astron. Astrofis., 36, 67

Raga, A. C., Velàzquez, P. F., Cantó, J., \& Masciadri, E. 2002, A\&A, 395, 647

Raymond, J. C. 1992, private communication

Rossi, P., Bodo, G., Massaglia, S., \& Ferrari, A. 1997, A\&A, 321, 672

Schmutzler, T., \& Tscharnutter, W. M. 1993, A\&A, 273, 318

Stafford, R. P., Bell, K. L., Hibbert, A., \& Wijesundera, W. P. 1994, MNRAS, 268,816

Sutherland, R. S., \& Dopita, M. A. 1993, ApJS, 88, 253

Sutherland, R. S., Bicknell, G. V., \& Dopita, M. A. 2003, ApJ, 591, 238

Voronov, G. S. 1997, Atom. Data Nucl. Data Tables, 65, 1

Wang, et al. 2001, ORNL/UGA Charge Transfer Database for Astrophysics, http://cfadc.phy.ornl.gov/astro/ps/data/ 